\title{
RESILIENCIA ACADÉMICA, NUEVAS PERSPECTIVAS DE INTERPRETACIÓN DEL APRENDIZAJE EN CONTEXTOS DE VULNERABILIDAD SOCIAL ${ }^{1}$
}

\author{
Gabriela Gómez ${ }^{2}$ \\ Marlene Rivas ${ }^{3}$
}

RESUMEN

Los países latinoamericanos tienen en común la persistente diferencia entre los resultados académicos de los estudiantes según su clase social. El presente ensayo se refiere a la relación entre el concepto de resiliencia y el aprendizaje en contextos de vulnerabilidad social. Se argumenta que este concepto permite comprender y analizar este fenómeno en su profundidad, de manera de contribuir a la búsqueda de mejores oportunidades de aprendizaje para todos. La promoción de la resiliencia académica resulta, en consecuencia, un objetivo clave para los sistemas educativos en vías de desarrollo. Junto con un recorrido por las definiciones más recientes de la resiliencia, se desarrolla una definición operativa del concepto para analizar el caso de la motivación y su relación con la comprensión lectora. Finalmente, se discute cómo fomentar la resiliencia en el medio escolar.

Palabras clave: comprensión lectora, motivación, resiliencia, vulnerabilidad.

\section{ACADEMIC RESILIENCE, NEW PERSPECTIVES OF INTERPRETING LEARNING IN CONTEXTS OF SOCIAL VULNERABILITY}

\section{ABSTRACT}

Latin American countries share the persistent difference in student academic results according to social class. This essay refers to the relationship between the concept of resilience and learning in contexts of social vulnerability. It is argued that this concept helps to understand and analyze this phenomenon in depth, to contribute to identifying better learning opportunities for all. A key objective for developing education systems is thus, the promotion of academic resilience. Along with a review of the most recent definitions of resilience, an operative definition of the concept is developed to analyze the case of motivation and its relation to reading comprehension. The essay concludes with a discussion on how to foster resilience in schools.

Keywords: motivation, reading comprehension, resilience, vulnerability.

1 Este ensayo forma parte del proyecto Fondecyt de Iniciación 11150209. Se agradece igualmente el financiamiento otorgado por el Proyecto Basal FB0003 del Programa de Investigación Asociativa de Conicyt.

2 Instituto de Ciencias de la Educación, Universidad de O'Higgins, Rancagua, Chile. Contacto: gabriela.gomez@uoh.cl

3 Centro de Investigación Avanzada en Educación, Universidad de Chile, Santiago, Chile. Contacto:marlene.rivas@ciae.uchile.cl 


\section{Antecedentes generales}

Las investigaciones internacionales que comparan la calidad de los sistemas educativos se han incrementado durante la última década. Diversos países de Latinoamérica han participado de evaluaciones estandarizadas como PISA, TIMSS, PIRLS y las mediciones promovidas por Unesco a través del Laboratorio Latinoamericano de Evaluación de la Calidad de la Educación (LLECE). Al mismo tiempo, la gran mayoría de los países del continente ha desarrollado evaluaciones nacionales tales como el Sistema Nacional de Evaluación de la Educación (SNEE) en México, el Programa de Evaluación de la Educación Básica Pruebas SABER o el Sistema Nacional de Medición de la Calidad Educativa (Simce) en Chile (Ferrer, 2006; Perassi, 2008).

Por otra parte, durante las últimas dos décadas, los sistemas educativos de la región han vivido importantes procesos de reformas, ajustes curriculares, organizacionales y estructurales. No obstante, los resultados de aprendizaje de la región aún se encuentran caracterizados por una gran falta de equidad. Esto se demuestra en que los resultados de las evaluaciones nacionales e internacionales ponen en relieve un problema clave de los sistemas educativos en Latinoamérica: en la región, existe una fuerte correlación entre las condiciones socioeconómicas de los estudiantes y sus resultados académicos. Los recientes resultados del Tercer Estudio Regional Comparativo y Explicativo, por ejemplo, dan cuenta de una correlación positiva entre los logros de aprendizaje y el PIB de cada país participante para todas las pruebas aplicadas (Treviño et al., 2015). El índice de nivel socioeconómico desarrollado ad hoc por el estudio muestra la misma tendencia.

En este contexto, dicha correlación afecta negativamente los logros de aprendizaje de los jóvenes y niños provenientes de los niveles socioeconómico más bajos. A la compleja situación que implica vivir en la pobreza, tener menor acceso a bienes básicos, salud y cultura, se agrega la baja calidad de la educación que estos niños están recibiendo durante su paso por la escuela. Lo anterior es confirmado por las evaluaciones mencionadas. Por ejemplo, para el caso de Chile, este es el país de mayor desigualdad de la región 
según el último informe LLECE (Treviño et al., 2015). A pesar de la mejora en los resultados de comprensión lectora en los estudios PISA, la distancia entre los niveles socioeconómicos se encuentra todavía alrededor de los 90 puntos, es decir, cerca de una desviación estándar del puntaje (Organisation for Economic Co-operation and Development, OECD, 2010). En el ámbito nacional, de acuerdo con la prueba Simce de comprensión de lectura para sexto año de primaria, la brecha entre los niveles socioeconómicos alto y bajo es equivalente o superior a la desviación estándar (Agencia de Calidad de la Educación, 2015). En resumen, a pesar de los esfuerzos en política pública en la región, los resultados indican que los estudiantes más vulnerables obtienen resultados significativamente más bajos que aquellos con mayores recursos. Pese a que en algunos países la brecha ha disminuido durante los últimos años, esta diferencia evidencia la elevada desigualdad predominante en los sistemas educativos latinoamericanos.

A estos antecedentes cabe agregar que, cuando se habla de vulnerabilidad, esta se refiere a condiciones que afectan no solo los resultados escolares de los niños, sino que también implica un menor acceso a la atención médica, cultura, recreación y, en general, a una menor calidad de vida (Yoshikawa, Aber y Beardslee, 2012). En la región, la pobreza afecta particularmente a las poblaciones rurales, minorías raciales, pueblos originarios y a los habitantes de los suburbios de las grandes ciudades.

Sin embargo, a pesar de las condiciones adversas que enfrentan en la escuela y su vida diaria, hay niños y niñas que crecen en la pobreza y, aun así, alcanzan resultados académicos iguales o superiores que sus pares que no se encuentran en situación de vulnerabilidad. Para distinguir a estos niños y niñas se puede hacer uso del concepto de resiliencia. Así, estos estudiantes que se sobreponen a las condiciones adversas de su entorno son los que la literatura especializada llama resilientes. Un aspecto clave subrayado por la literatura reciente acerca de la resiliencia es que estos casos no son accidentales, aislados, ni tampoco el resultado de un temperamento psicológico extraordinario. La resiliencia es un proceso relacionado con múltiples factores y que, en consecuencia, puede ser intervenida y fomentada. Se trata, por lo 
tanto, de una prueba clara de que es posible mejorar los logros de aprendizaje de quienes viven en contextos de vulnerabilidad.

La investigación se propone ahondar en el concepto de resiliencia, resiliencia académica y, específicamente, la resiliencia académica en comprensión lectora. Esta lógica argumental permitirá indagar cómo esta puede ser un medio para desarrollar un mejor conocimiento de la realidad de la educación en contextos de vulnerabilidad. En las siguientes páginas se argumenta la necesidad de desarrollar investigación y propuestas pedagógicas que aborden la relación entre la comprensión lectora y la resiliencia. Para ello, se describirán dos conceptos clave del marco teórico de la resiliencia: los factores de riesgo y los factores de protección. Además, se desarrollará una descripción de aquellos factores que determinan la comprensión lectora, su importancia y su relación con la resiliencia. Todo ello permitirá elaborar una posible operacionalización del concepto y discutir con mayor profundidad sus posibles aplicaciones pedagógicas. En síntesis, el tema central de este ensayo es identificar con exactitud los mecanismos que determinan la resiliencia, de manera de fomentarla.

De acuerdo con ello, nos hemos basado en la premisa de que existen factores relacionados, tanto con la resiliencia como con la comprensión lectora y que establecen diferencias entre los estudiantes resilientes y el resto de los niños que comparten su situación de vulnerabilidad. En relación con este último punto es necesario discutir el nivel de aleatoriedad de la resiliencia. ¿Se trata simplemente de la consecuencia de una habilidad cognitiva superior que es inherente a algunos niños y niñas? La literatura entrega evidencia acerca de un aspecto clave: existen factores determinantes de la resiliencia que la fortalecen o disminuyen, estos son llamados factores protectores y factores de riesgo. Los primeros, protegen a los estudiantes vulnerables de las amenazas de su entorno y se relacionan con un mejor rendimiento académico. Los segundos, se suman a la vulnerabilidad y aumentan la probabilidad de obtener malos resultados académicos. Una parte de estos factores son individuales y están vinculados con las actitudes, competencias y recursos con los que cuentan los estudiantes. Otra parte se encuentra determinada 
por las familias y las escuelas. Los padres, parientes e instituciones educativas pueden actuar como figuras de protección y mecanismos contra la condición o, por el contrario, agravar y añadir mayores dificultades.

Factores protectores individuales pueden ser las motivaciones, un hogar donde se fomenta la lectura y la presencia de figuras modélicas. A nivel de la escuela, se pueden mencionar como factores protectores un entorno favorable, el compromiso de los profesores con los logros de los estudiantes o las estructuras comunitarias. La influencia de actores e instituciones puede funcionar en dos sentidos: ya sea neutralizando los factores de riesgo que aumentan la probabilidad de un rendimiento deficiente, o bien, fomentando los factores protectores, los cuales aumentan las probabilidades de un rendimiento destacado. Además, se presume que es posible controlar estos factores con el fin de generar y fomentar reacciones de resiliencia.

\section{Resiliencia y vulnerabilidad}

En la literatura, la resiliencia se entiende como una reacción positiva, un desarrollo constructivo o dinámico al enfrentarse a una catástrofe, amenaza o situación estresante (Cicchetti, 2013; Hanewald, 2011; Masten, 2014); como combinación entre experiencias que suponen un riesgo y resultados positivos desde el punto de vista psicológico (Rutter, 2006).

Existen dos corrientes principales respecto del tema: la primera de ellas, perteneciente a las décadas de los setenta y ochenta y representa la etapa de desarrollo del concepto. Durante este periodo, la definición enfatizó las particularidades y la personalidad de los resilientes, comprendiendo el concepto como una característica natural, un rasgo propio de ciertos individuos (Hanewald, 2011; Werner, 1996). Actualmente, las nuevas corrientes destacan el hecho de que la resiliencia puede ser desarrollada como un proceso dinámico y no estático (Kolar, 2011; Masten y Obradovic, 2008; Rutter, 2006). Como señala Ciccheti (2010): "Resilience is not something an individual "has": it is a multiply determined 
developmental process that is not fixed or immutable" (p. 146). Investigaciones recientes destacan la reacción resiliente frente a la adversidad como un constructo multidimensional que implica la combinación de procesos psicológicos, socioculturales y biológicos (Hughes, 2012; McEwen, Gray \& Nasca, 2015).

En el ámbito educativo, el concepto de resiliencia ha sido utilizado para describir diferentes fenómenos, tales como la adaptación a la escuela de los estudiantes pertenecientes a minorías étnicas, víctimas de abuso, o que han vivido situaciones traumáticas como la muerte de sus padres, guerra o desastres naturales (Fluxá y Acosta, 2009). En educación, una de las formas para acercarse al estudio de la resiliencia es la denominada resiliencia académica (Gordon Rouse, 2001). En estos casos, los estudiantes resilientes tienen como característica la conjugación de resultados académicos mejores a lo esperado y ciertas condiciones contextuales de riesgo. El riesgo, a su vez, es definido como la probabilidad de que el estudiante obtenga un rendimiento normal o sobresaliente, en ciertas condiciones de estrés o trauma como las mencionadas con anterioridad (Masten $\&$ Obradovic, 2008). En este caso, si los estudiantes superan el riesgo y logran resultados académicos normales, se dice que tienen una reacción resiliente positiva académicamente. En el segundo, si los estudiantes logran resultados por sobre la norma, se dirá que la reacción no solo es positiva, sino que sobresaliente (Cappella \& Weinstein, 2001).

Desde esta perspectiva, el proceso resiliente es caracterizado por la interacción de factores opuestos: factores de riesgo y protección que participan en el desarrollo de una reacción resiliente (Cappella \& Weinstein, 2001). Los factores de riesgo incrementarán las posibilidades de obtener resultados deficientes (Evans, Li \& Whipple, 2013). El riesgo se incrementará mientras más factores acumule; por ejemplo, vivir en la pobreza es, en sí misma, una situación de riesgo, pero si además de esto existe un ambiente desprotegido, violencia en la escuela o problemas de salud, la

4 En español: La resiliencia no es algo que un individuo "tiene": es un proceso de desarrollo de múltiples causas que no es fijo ni inmutable. 
situación empeora (Catterall, 1998; Evans et al., 2013). Por otro lado, los factores de protección son un grupo de características que refuerzan y fomentan la resiliencia: "características, factores o situaciones que son endógenas a las personas o sus ambientes e incrementan su capacidad para enfrentar la adversidad o disminuir las posibilidades de desarrollar desajuste psicosocial a pesar de los factores de riesgo" (Fluxá y Acosta, 2009, pp. 24-25). Los factores de protección son considerados una condición obligatoria para que el proceso de resiliencia se lleve a cabo. Los factores protectores y de riesgo pueden ser individuales, familiares o sociales (Dyer \& McGuinness, 1996; Evans et al., 2013).

En resumen, como propone Kolar (2011), para explicar un determinado fenómeno utilizando provechosamente el concepto de resiliencia, es necesario definir tres elementos con precisión: condiciones de riesgo, indicador de resiliencia (resultados) y los factores involucrados en el proceso. Masten y Obradovic (2008) distinguen tres niveles de resiliencia según un indicador de logro preestablecido: a) niveles de logro más altos que los esperados para determinado grupo; b) la capacidad de mantener un desempeño regular a pesar de la adversidad; c) recuperar un nivel normal de logro después de un evento traumático.

Si se aplican estas estrategias de análisis en los casos de estudiantes provenientes de familias de bajos ingresos y con buenos resultados escolares, es posible postular que, de un gran número de niños vulnerables, una parte de ellos tendrá resultados iguales o superiores a lo esperado, es decir, serán resilientes. Esta condición estará determinada por factores que protegen a los individuos de los riesgos, así como por factores que los incrementan.

Centrándose en las proporciones de individuos resilientes y no resilientes que se pueden encontrar en un grupo dado, la literatura muestra que la resiliencia es un fenómeno escaso, pero persistente. Desde la investigación, Grotberg $(1995 ; 2006)$ concluye que cuando se enfrenta un evento traumático, un tercio de las víctimas desarrolla conductas resilientes. La misma tendencia ha quedado comprobada por Werner (1996) en uno de los estudios clásicos en la materia 
(Vulnerable but invincible: High risk children from birth to adulthood); allí se demostró que, de un grupo de niños en riesgo, solo un tercio de ellos logra una adultez normal. La resiliencia académica resulta ser menos frecuente para Capella y Weinsten (2001), donde solo un 15\% de aquellos que tienen resultados deficientes en educación primaria mejora su rendimiento en secundaria. Algunas investigaciones (Gómez, Valenzuela y Sotomayor, 2015) han permitido confirmar este patrón: un tercio de los estudiantes chilenos pertenecientes al cuarto percentil más pobre de los participantes de la prueba PISA 2009, alcanzó resultados iguales o superiores a la media nacional en el test de comprensión lectora.

\section{Factores protectores y de riesgo a nivel de los sujetos y de la comunidad escolar}

En cuanto a los atributos personales y psicológicos de los individuos, serán factores protectores la autoimagen que tienen los estudiantes y su motivación e involucramiento con los contenidos (Gordon Rouse, 2001). Dyer y McGuinness (1996) también mencionan como factores de protección la autoimagen que el sujeto tenga de sí mismo, en tanto que Martin y Marsh (2006) proponen un modelo llamado 5-C, afirmando que la resiliencia depende de cinco factores: seguridad (autoeficacia), coordinación (planificación), control, compostura (baja ansiedad) y compromiso (persistencia).

Además de la pertenencia a un estrato socioeconómico bajo, existen otras características etnográficas que pueden implicar el riesgo de obtener resultados académicos insuficientes. Si además de vivir en la pobreza, los niños pertenecen a una minoría o a una familia monoparental, el riesgo aumenta (Cappella $\&$ Weinstein, 2001). Otra consecuencia de pertenecer a una minoría que incrementa el riesgo es enfrentar experiencias de violencia y racismo (Grotberg, 2006). Un dato interesante de relevar es que estudios como el de Evans y colaboradores (2013) señalan que una de las características que refuerza la resiliencia académica es pertenecer al género femenino.

Respecto de la vida familiar, un ambiente tranquilo, el respaldo y estímulo de los padres y familiares que sean modelos a seguir y 
buenas relaciones con sus pares pueden ser igualmente favorables; también se mencionan como factores favorables la existencia de un sistema de valores, ya sea que se traten de creencias religiosas o éticas. En este sentido, Neil (2006) describe la resiliencia familiar como "one that has the capacity of continue growing in spite of adversity and maintain a coherence in the way they confront critical situations ${ }^{5 "}$. La literatura destaca la importancia de la presencia de un adulto significativo (Ciccheti, 2010), una figura modelo que puede ser parte de la familia, un profesor, un profesional de la salud u otro. Estos mentores o cuidadores tienen el poder de reforzar la autoestima de quienes se encuentran en situaciones de vulnerabilidad y ayudarlos a tomar sentido sobre eventos traumáticos que han vivido (Tisseron, 2009).

Los posibles factores que refuerzan la resiliencia en la escuela son: un ambiente positivo y regulado, actitudes positivas de los pares hacia la vida académica y su disciplina (Cappella $\&$ Weinstein, 2001). Tisseron (2009) menciona al respecto como un factor positivo e importante el que exista otro tipo de organizaciones sociales, además de la escuela, que entreguen soporte (servicios de salud, organizaciones sociales y comunitarias). En relación con la escuela en particular, el autor identifica tres características destacadas por la literatura: en primer lugar, un ambiente favorable sin violencia; las expectativas de la escuela acerca de los estudiantes; y tercero, que la escuela permita a los estudiantes sentirse útiles y que se puedan expresar a través de diversas actividades colectivas, curriculares o extracurriculares, que sean desarrolladas en los centros educacionales.

La conjugación de estos factores en una comunidad educativa necesita ser analizada entre los distintos niveles de participantes que operan en y a través de ella, a fin de entender el fenómeno de la resiliencia inserto dentro de dinámicas relacionales y ecológicas de la escuela (Bronfenbrenner, 1979). Estas dinámicas guardan relación con el clima de los espacios de aprendizaje, la convivencia favorable,

En español: Individuos que tienen la capacidad de continuar creciendo a pesar de la adversidad y mantener una coherencia en el modo en que enfrentan situaciones críticas. 
sin violencia, así como con aspectos del entorno de las expectativas docentes y de las políticas de las comunidades educativas.

Respecto del ambiente favorable, este se relaciona con los estudios acerca del clima en el aula y la convivencia escolar en las comunidades educativas. Para el caso chileno, estas temáticas se han desarrollado principalmente desde estudios de violencia y bullying escolar (López, Bilbao y Rodríguez, 2012). Este tema ha adquirido relevancia como factor para el buen desempeño de los niños y niñas en Chile desde la Política de Convivencia Escolar (Ministerio de Educación de Chile, Mineduc, 2010). Desde esta perspectiva, la escuela debiese ser un espacio donde los niños construyan aprendizajes académicos y socioemocionales y aprendan a convivir de manera democrática, convirtiéndose en los protagonistas de sociedades más justas y participativas (López, 2014). En estudiantes resilientes provenientes de situaciones de vulnerabilidad el factor protector de la escuela que genera un buen clima escolar es estructural para apoyar un exitoso rendimiento académico (Dearden, 2004).

En cuanto a las expectativas de la escuela en relación con los estudiantes, los profesores poseen un rol clave dentro de la comunidad educativa para apoyar y potenciar el proceso de resiliencia (Truebridge, 2016). Las creencias de los docentes se forman a partir de sus experiencias y dan cuenta de la influencia de diversos esquemas culturales (Makuc, 2008). Así mismo, las expectativas y creencias de los docentes hacia los estudiantes impactan tanto en el resultado que estos obtienen como en la interacción en el aula. Henderson y Milstein (2010), por ejemplo, identifican las expectativas elevadas de los docentes como un paso importante para promover la resiliencia en las escuelas, en tanto que Gómez y colaboradores (2016) señalan que las expectativas y creencias de los docentes inciden en diversos niveles del desarrollo del aprendizaje.

Finalmente, para la promoción de la resiliencia es importante la inclusión democrática de los estudiantes y sus familias en las comunidades educativas, lo que implica brindarles oportunidades de participación significativas. Para Henderson y Milstein (2010) los estudiantes son recursos y no objetos o problemas pasivos de 
la escuela. Desde esta óptica es necesario, entonces, promover relaciones de confianza y respeto mutuo entre el equipo directivo, de gestión y los docentes, a fin de promover una gestión democrática del profesorado y los profesionales de apoyo psicosocial (Sánchez et al., 2011). Sobre esta base la escuela puede entregar experiencias de participación significativa para los estudiantes y sus familias, de modo que se sientan útiles e involucrados.

\section{Comprensión lectora y motivación}

Como previamente se mencionó, la resiliencia se verá afectada por la relación que los individuos tienen con el contenido académico. Para profundizar este análisis nos referiremos al caso de los libros y la lectura, ya que al igual que la resiliencia, el proceso de lectura compromete aspectos cognitivos y no cognitivos.

Respecto de la comprensión lectora existe abundante literatura en torno a los posibles factores involucrados en su adquisición, relacionados principalmente con el aprendizaje temprano y los primeros años de educación formal (Snowling \& Hulme, 2005). Sin embargo, no existen muchas investigaciones acerca del desarrollo de la comprensión una vez terminado el periodo de enseñanza obligatoria. En este contexto, destaca el estudio del llamado compromiso lector (Reading engagement) (Guthrie et al., 2007; Taboada, Tonks, Wigfield \& Guthrie, 2009). Este concepto es utilizado para referirse a factores relacionados con las actitudes, las estrategias de lectura, la motivación y sus interacciones: "Engaged readers are viewed as motivated, strategic, knowledgeable, and socially interactive. They read widely for a variety of purposes and capitalize on situations having potential to extend literacy"6 (Baker, Afflerbach \& Reiking, 1996, p. XIII). De este modo, esta propuesta subraya la importancia de tomar en consideración no solo el proceso cognitivo, sino también los aspectos subjetivos que se ponen en juego a la hora de abordar un texto.

6 En español: Lectores comprometidos son vistos como motivados, estratégicos, e interactivos socialmente. Son capaces de leer en función de variados propósitos y capitalizar situaciones que tienen el potencial de expandir la literacidad. 
Desde el punto de vista de la resiliencia, estos elementos no cognitivos son un componente clave, ya que considera factores que describen lo que los individuos aportan subjetivamente al proceso de comprensión. En este sentido, la motivación se encuentra directamente relacionada con la base del proceso de desarrollo de la comprensión (Wigfield, Gladstone \& Turci, 2016). La interrelación entre motivación y comprensión de lectura se opone a la relación de los factores económicos (tal como la pobreza) con la lectura, ya que estos últimos son generales y determinan el conjunto total del proceso escolar e incluso otros aspectos sociales, como la salud o la integración laboral.

La motivación, por el contrario, es directamente dependiente de la relación que el lector sostiene con la lectura, de lo que siente y piensa respecto de esta y de los libros. La motivación se manifiesta por medio de un conjunto de creencias, valores y metas que se atribuyen a la acción de leer (Wigfield et al., 2016).

Respecto de las creencias, estas se expresan en la confianza del sujeto en su propia capacidad como lector (autoeficacia), en su autonomía y su capacidad de autorregulación. La autoeficacia describe la imagen que los lectores tienen acerca de sí mismos, en relación con las interacciones con los libros y la lectura. Como señalan Marsh y Martin (2011), generar actitudes positivas que mejoren la imagen que los estudiantes tienen de sus propias habilidades debería ser uno de los objetivos principales de cualquier sistema educacional, porque las actitudes positivas y los resultados escolares se fortalecen mutuamente. Respecto de la autorregulación, Alonso Tapia (2005) destaca la importancia de la supervisión y la autorregulación de la lectura para una profunda comprensión del texto. Para este autor, el control es uno de los factores que explican las diferencias individuales, este puede ser enseñado y también puede ser objeto de "entrenamiento explícito" (p. 79). Al mismo tiempo, el autor menciona la probable relación entre la habilidad para controlar la comprensión de lectura y la de mantener el interés. Por el contrario, no conocer la necesidad de regular la comprensión afectaría la calidad de la misma de un modo negativo. 
Los valores positivos que el lector asocia con la lectura se traducen en la importancia dada a la actividad; para desarrollar ese interés se suele apelar a los conceptos de motivación extrínseca e intrínseca. La primera está relacionada con recompensas sociales, tales como las evaluaciones o el reconocimiento de otros respecto de la lectura, en tanto que la segunda se refiere al propio interés declarado, también conocido como el gusto por la lectura. Es importante destacar que el valor dado a la lectura puede variar y, de hecho, tiende a disminuir en la adolescencia como parte del proceso de autoconstrucción del sujeto. Durante los primeros años de educación formal, los niños suelen abrumar a sus profesores y padres con su curiosidad natural. La literatura confirma que este interés por la disciplina tiende a decrecer, alcanzando su punto crítico durante la adolescencia (Córdova \& Lepper, 1996). Evitar este decrecimiento es un objetivo pedagógico fundamental si se quiere considerar la interdependencia entre motivación, valoración y comprensión lectora. Como se señalaba con anterioridad, la literatura actual destaca que estos factores se fortalecen unos con otros (Valentine y DuBois, 2005). Respecto de las metas, estas se refieren a la pregunta de para qué se lee; entre las respuestas que tienen relación positiva con la comprensión lectora (Guthrie \& Klauda, 2014; Wigfield et al., 2016) está el interés por aumentar el propio conocimiento ("leo porque me interesa un tema determinado"), el aprendizaje ("leo porque valoro el aprender") o el ser mejor que los demás ("leo para saber más que mis compañeros").

Los factores que permiten describir los diferentes aspectos de la motivación coinciden en gran medida con los factores protectores vinculados con la resiliencia. De esta manera, permiten pensar de una forma más operativa cómo fomentar la resiliencia por medio de la motivación a la comprensión lectora.

\section{Propuestas para la investigación educativa}

Para la investigación en educación en Latinoamérica es clave abordar la pregunta acerca de cómo viven y cómo son las experiencias escolares de los niños que crecen en la pobreza y cómo logran 
alcanzar altos resultados académicos a pesar de que las condiciones de adversidad en las que se encuentran cotidianamente. Alcanzar una comprensión profunda del complejo proceso de relación entre los resultados académicos y el nivel socioeconómico puede lograrse si se analizan en forma combinada elementos cognitivos y no cognitivos. En este contexto, al observar con más detalle los resultados evaluativos de los estudiantes en contextos de vulnerabilidad, se puede apreciar la escasa homogeneidad en este grupo. Particularmente, se destaca la existencia de ciertos estudiantes que, a pesar de provenir de un grupo de riesgo, obtienen altos resultados. Este fenómeno, contrario a la correlación entre nivel socioeconómico y resultados académicos, es identificado en la literatura especializada como resiliencia académica.

Se ha profundizado la descripción del caso específico de la resiliencia académica en comprensión lectora como un medio para identificar acciones concretas que contribuyan al fomento de la resiliencia. Estos antecedentes han permitido observar la relación entre la resiliencia y la motivación por el compromiso lector, y cómo ambos confluyen en los mecanismos que subyacen a una reacción resiliente. Lo que se concluye de este caso de resiliencia es la importancia de considerar la interacción de múltiples factores para la generación de una reacción resiliente.

La literatura señala que la resiliencia es un fenómeno favorable para el aprendizaje y, en consecuencia, es deseable promoverla en el sistema educacional. El análisis de casos de estudiantes vulnerables que alcanzan resultados destacados es fundamental, ya que ellos, los niños resilientes, tienen la clave para mejorar la calidad de la educación para los que más lo necesitan. Para ello, es necesario determinar la adecuada intervención de los mecanismos que la desarrollan, a partir de la idea de que la resiliencia puede ser aprendida (Kotliarenco, Cáceres y Fontecilla, 1997).

Así mismo, se puede considerar a los estudiantes vulnerables con excelente rendimiento académico como un modelo para el resto de sus pares y, a través de su experiencia, contribuir al desarrollo de un sistema educativo más equitativo. La promoción de la resiliencia 
dentro del sistema escolar es un objetivo concreto y alcanzable. Identificar con exactitud los mecanismos que la determinan y cómo fomentarlos ha sido el objetivo de estas páginas.

\section{Referencias}

Agencia de Calidad de la Educación, ACE. (2015). Resultados educativos 2015. Santiago de Chile: Mineduc.

Baker, L., Afflerbach, P., \& Reinking, D. (1996). Developing engaged readers in school and home communities. Mahwah, N.J.: Lawrence Erlbaum.

Bronfenbrenner, U. (1979). The ecology of human development. Cambridge, MA: Harvard University Press.

Cappella, E. \& Weinstein, R. S. (2001). Turning around reading achievement: Predictors of high school students' academic resilience. Journal of Educational Psychology, 93(4), 758.

https://doi.org/10.1037//0022-0663.93.4.758

Catterall, J. S. (1998). Risk and resilience in student transitions to high school. American Journal of Education, 106(2), 302-333.

https://doi.org/10.1086/444184

Cicchetti, D. (2010). Resilience under conditions of extreme stress: A multilevel perspective. World Psychiatry, 9(3), 145-154. https://doi.org/10.1002/j.2051-5545.2010.tb00297.x

Cicchetti, D. (2013). Annual research review: Resilient functioning in maltreated children-past, present, and future perspectives. Journal of Child Psychology and Psychiatry, 54(4), 402-422. https://doi.org/10.1111/j.1469-7610.2012.02608.x

Cordova, D. \& Lepper, M. (1996). Intrinsic motivation and the process of learning: Beneficial effects of contextualization, personalization, and choice. Journal of Educational Psychology, 88(4), 715-730. https://doi.org/10.1037//0022-0663.88.4.715

Dearden, J. (2004). Resilience: A study of risk and protective factors from the perspective of young people with experience of local authority care. Support Learning, 19(4), 187-193. https://doi.org/10.1111/j.0268-2141.2004.00346.x

Dyer, J. \& McGuinness, T. (1996). Resilience: Analysis of the concept. Archives of Psychiatric Nursing, 10(5), 276-282.

https://doi.org/10.1016/s0883-9417(96)80036-7 
Evans, G. W., Li, D., \& Whipple, S. S. (2013). Cumulative risk and child development. Psychological Bulletin, 139(6), 1342-1396.

https://doi.org/10.1037/a0031808

Ferrer, G. (2006). Estado de situación de los sistemas nacionales de evaluación de logros de aprendizaje en América latina. Programa para la Promoción de la Reforma Educativa en América Latina y el Caribe, PREAL. Recuperado de

http://enlaceacademico.ucr.ac.cr/sites/default/files/publicaciones/ Libro\%20completo.pdf

Fluxá, F. y Acosta, E. (2009). Conflicto y resiliencia en contextos educativos. Santiago de Chile: UMCE.

Gómez, G., Sotomayor, C., Jéldrez, E., Bedwell, P., Domínguez, A. M., Ávila, N., y Calderón, M. (2016). La producción escrita de estudiantes y escuela en base a resultados Simce, factores contextuales y modelos de buenas prácticas docentes. Informe final proyecto F911437 FONIDE-Mineduc. Santiago de Chile.

Gómez, G., Valenzuela, J. P., \& Sotomayor, C. (2015). Against all odds: Outstanding reading performance among Chilean youth in vulnerable conditions. Comparative Education Review, 59(4) 693-716. https://doi.org/10.1086/683108

Gordon Rouse, K. A. (2001). Resilient students' goals and motivation. Journal of Adolescence, 24(4), 461-472.

https://doi.org/10.1006/jado.2001.0383

Grotberg, E. H. (1995). The international resilience project: Promoting resilience in children. Arlington, VA: University of Alabama at Birmingham. Civitan International Research Center.

Grotberg, E. H. (2006). La resiliencia en el mundo de hoy: cómo superar las adversidades. Barcelona: Gedisa.

Guthrie, J. T., Hoa, A. L. W., Wigfield, A., Tonks, S. M., Humenick, N. M., $\&$ Littles, E. (2007). Reading motivation and reading comprehension growth in the later elementary years. Contemporary Educational Psychology, 32(3), 282-313.

http://dx.doi.org/10.1016/j.cedpsych.2006.05.004

Guthrie, J. T. \& Klauda, S. L. (2014). Effects of classroom practices on reading comprehension, engagement, and motivations for adolescents. Reading Research Quarterly, 49(4), 387-416.

https://doi.org/10.1002/rrq.81

Hanewald, R. (2011). Reviewing the literature on "at-risk" and resilient children and young people. Australian Journal of Teacher Education, 36(2), 16-29. https://doi.org/10.14221/ajte.2011v36n2.2 
Henderson, N. y Milstein, M. (2010). Resiliencia en la escuela. Buenos Aires: Paidós.

Hughes, V. (2012). The roots of resilience. Nature, 490(7419), 165-167. https://doi.org/10.1038/490165a

Kolar, K. (2011). Resilience: Revisiting the concept and its utility for social research. International Journal of Mental Health and Addiction, 9(4), 421433.

https://doi.org/10.1007/s11469-011-9329-2

Kotliarenco, M., Cáceres, I., y Fontecilla, M. (1997). Estado de arte en resiliencia. Washington, DC: Organización Panamericana de la Salud.

López, V. (2014). Convivencia escolar. Apuntes educación y desarrollo Post2015. Unesco $n^{\circ} 4$. Recuperado de

http://www.unesco.org/fileadmin/MULTIMEDIA/FIELD/Santiago/pdf/ APUNTE04-ESP.pdf

López, V., Bilbao, M. D. L. A., y Rodríguez, J. I. (2012). La sala de clases sí importa: incidencia del clima de aula sobre la percepción de intimidación y victimización entre escolares. Universitas Psychologica, 11(1). Recuperado de

https://www.psiucv.cl/wp-content/uploads/2014/01/La-sala-de-clasessí-importa.pdf

Marsh, H. W. \& Martin, A. J. (2011). Academic self-concept and academic achievement: Relations and causal ordering. British Journal of Educational Psychology, 81(1), 59-77.

https://doi.org/10.1348/000709910x503501

Martin, A. J. \& Marsh, H. W. (2006). Academic resilience and its psychological and educational correlates: A construct validity approach. Psychology in the Schools, 43(3), 267-281.

https://doi.org/10.1002/pits.20149

Masten, A. S. (2014). Global perspectives on resilience in children and youth. Child development, 85(1), 6-20.

https://doi.org/10.1111/cdev.12205

Masten, A. S. \& Obradovic, J. (2008). Disaster preparation and recovery: Lessons from research on resilience in human development. Ecology and Society, 13(1), 1-16. https://doi.org/10.5751/es-02282-130109

McEwen, B. S., Gray, J. D., \& Nasca, C. (2015). Recognizing resilience: Learning from the effects of stress on the brain. Neurobiology of stress, $1,1-11$. https://doi.org/10.1016/j.ynstr.2014.09.001 
Ministerio de Educación de Chile, Mineduc. (2010). Politica de convivencia escolar. Santiago de Chile: Autor.

Makuc, M. (2008). Teorías implícitas de los profesores acerca de la comprensión de textos. Revista signos, 41(68), 403-422.

Neil, S. E. S. (2006). Intensificar la resiliencia en el grupo familiar: un enfoque transgeneracional hacia el cambio positivo en las familias disfuncionales. En E. H. Grotberg (Ed.), La resiliencia en el mundo de hoy: cómo superar las adversidades (pp. 91-129). Madrid: Gedisa.

Organisation for Economic Co-operation and Development, OECD (Ed.). (2010). Pisa 2009 results: Overcoming social background-equity in learning opportunities and outcomes (volume II). Paris: Autor.

Perassi, Z. (2008). La evaluación en educación: un campo de controversias. San Luis, Argentina: Ediciones LAE.

Rutter, M. (2006). Implications of resilience concepts for scientific understanding. Annals of the New York Academy of Sciences, 1094(1), $1-12$. https://doi.org/10.1196/annals.1376.002

Sánchez, L., Carvajal, M., Huerta, M., Ahumada, F., Henríquez, A., Murúa, M., ... y Rojas, G. (2011). Aprender de la experiencia: indagando juntos buenas prácticas en convivencia escolar. Mejoramiento escolar en acción, 115-208. Recuperado de

http://static.convivencia.psiucv.cl/wp-content/uploads/2012/08/ Sanchez-et-al-Indagando-juntos-buenas-prácticas-en-convivenciaescolar.pdf

Snowling, M. J. \& Hulme, C. (2005). Editorial part IV. En M. J. Snowling $\&$ C. Hulme (Eds.), The science of reading: A handbook (pp. 269-271). London: Blackwell.

Taboada, A., Tonks, S., Wigfield, A., \& Guthrie, J. T. (2009). Effects of motivational and cognitive variables on reading comprehension. Reading and Writing, 22(1), 85-106.

https://doi.org/10.1007/s11145-008-9133-y

Tapia, J. A. (2006). Motivar en la escuela, motivar en la familia. Madrid: Ediciones Marmota.

Tisseron, S. (2009). La résilience. France: PUF.

Treviño, E., Fraser, P., Meyer, A., Morawietz, L., Inostroza, P., y Naranjo, E. (2015). Informe de resultados TERCE: Factores asociados. Santiago de Chile: LLECE. 
Truebridge, S. (2016). Resilience: It begins with beliefs. Kappa Delta Pi Record, 52(1), 22-27.

Valentine, J. \& DuBois, D. (2005). Effects of self-beliefs on academic achievement and vice versa. En H. W. Marsh, R. G. Craven, \& D. M. McInerney (Eds.), The new frontiers of self-research (pp. 53-77). Greenwich, CT: Information Age Publishing.

Werner, E. E. (1996). Vulnerable but invincible: High risk children from birth to adulthood. European Child \& Adolescent Psychiatry, 5(S1), 47-51. https://doi.org/10.1007/bf00538544

Wigfield, A., Gladstone, J. R., \& Turci, L. (2016). Beyond cognition: Reading motivation and reading comprehension. Child Development Perspectives, 10(3), 190-195.

https://doi.org/10.1111/cdep.12184

Yoshikawa, H., Aber, J. L., \& Beardslee, W. R. (2012). The effects of poverty on the mental, emotional, and behavioral health of children and youth: Implications for prevention. American Psychologist, 67(4), 272-284. https://doi.org/10.1037/a0028015

Recibido: 10/11/2016

Aceptado: 07/08/2017 